\title{
Role of Active Movement in Place-Specific Firing of Hippocampal Neurons
}

\author{
Eun Young Song, ${ }^{1}$ Yun Bok Kim, ${ }^{1}$ Young Ho Kim, ${ }^{2}$ and Min Whan Jung ${ }^{1 *}$
}

\begin{abstract}
The extent of external and internal factors contributing to location-specific firing of hippocampal place cells is currently unclear. We investigated the role of active movement in location-specific firing by comparing spatial firing patterns of hippocampal neurons, while rats either ran freely or rode a motorized cart on the same circular track. Most neurons changed their spatial firing patterns across the two navigation conditions ("remapping"), and they were stably maintained across repeated active or passive navigation sessions. These results show that active movement is a critical factor in determining place-specific firing of hippocampal neurons. This could explain why passive displacement is not an effective way of acquiring spatial knowledge for subsequent active navigation in an unfamiliar environment. $\odot 2004$ Wiley-Liss, Inc.
\end{abstract}

KEY WORDS: hippocampus; place cell; active navigation; passive navigation; remapping

\section{INTRODUCTION}

One of the most striking and consistent features of hippocampal neuronal activity is place-specific firing. Principal cells of the hippocampus fire at elevated rates when an animal is within restricted places of a given environment but are silent elsewhere (O'Keefe and Dostrovsky, 1971; Jung and McNaughton, 1993). These "place cells" are considered to represent allocentric spatial information (O'Keefe and Nadel, 1978). Rotation of distal visual cues induces corresponding rotation of place fields (e.g., O'Keefe and Conway, 1978), demonstrating the role of external sensory inputs in determining place cell activity. However, place-specific firing can be maintained following removal of all distal visual cues (Muller and Kubie, 1987; O'Keefe and Speakman, 1987) or turning off the light (Quirk et al., 1990; Markus et al., 1994), indicating that place-specific firing can also be maintained by summing internally generated displacement cues (path integration; but see Save et al., 2000). Currently, the relative contributions of external and internal information to the discharge of hippocampal neurons are not fully understood.

We investigated the role of active movement in place-specific firing of hippocampal neurons. In a previous study that compared hippocampal neuronal activity under active and passive navigation conditions, place cells stopped firing in the place field when rats were returned there while physi-

\footnotetext{
${ }^{1}$ Neuroscience Laboratory, Institute for Medical Sciences, Ajou University School of Medicine, Suwon, Korea; ${ }^{2}$ Digital Biotech Corporation, Ansan, Korea

Grant sponsor: Ministry of Health and Welfare; Grant number: 02-PJ2-PG6DC04-0001.

*Correspondence to: Min Whan Jung, Neuroscience Laboratory, Institute for Medical Sciences, Ajou University, Suwon 443-721, Korea.

E-mail: min@ajou.ac.kr

Accepted for publication 2 April 2004

DOI 10.1002/hipo.20023

Published online in Wiley InterScience (www.interscience.wiley.com).
}

cally restrained (Foster et al., 1989). This study leaves open the possibility that hippocampal neurons may actively discharge during passive movement if rats are not physically restrained. Indeed, a more recent study has shown that place cells do not shut off under a less restrained condition. In this study, rats were passively moved on a mobile robot with their heads fixed to a robot with the body suspended in a hammock with legs dangling freely. Hippocampal neurons showed spatially selective firing patterns, although they were broadly tuned (Gavrilov et al., 1998). This study did not compare active versus passive navigation, thus it is difficult to determine whether hippocampal neurons maintain or alter their firing patterns across the two navigation conditions. In the present study, we compared firing patterns of hippocampal neurons across active and passive navigation conditions in the same environment with minimal physical restraint. If place-specific firing of hippocampal neurons is constructed primarily from external sensory inputs and path integration, similar patterns of neuronal activity would be expected across the two navigation conditions. Otherwise, if factors such as motor command signals and efference copy play important roles, different spatial firing patterns will be observed. The present study has been presented previously in abstract form (Song and Jung, 2002).

\section{MATERIALS AND METHODS}

\section{Behavior}

Fifteen male Sprague-Dawley rats (280-320 g, 8-11 weeks old) were trained to (1) actively run, and (2) passively ride a motorized cart $(16 \mathrm{~cm}$ long, $8 \mathrm{~cm}$ wide, 4.5 $\mathrm{cm}$ above the maze floor with a 5-cm-high wall) on a circular track to obtain water reward on two locations $\left(180^{\circ}\right.$ apart; identical locations across active and passive navigation sessions). Movement of the cart was remotely controlled by an experimenter, and the animals stopped completely at the reward sites in both navigation conditions. The delivery of water was automatically controlled in active navigation sessions. As the rat approached one of the reward locations, a photodetector activated solenoid valves so that $50 \mu \mathrm{l}$ of water was dropped from polyethylene tubing to the maze floor at both reward locations. Water delivery was completed before arrival of the animal at the reward locations. In passive navigation sessions, the 
same volume $(50 \mu \mathrm{l})$ of water was manually delivered using a syringe onto a small transparent acryl plate that was attached to the front side of the motorized cart at the reward locations. The circular track was $100 \mathrm{~cm}$ in diameter (OD), and the width of the track was $15 \mathrm{~cm}$. It was elevated $(40 \mathrm{~cm})$ from the floor with $5 \mathrm{~cm}$ high walls along the entire track. A 5-cm-wide rail was installed on the maze floor along which the cart was driven. The recording room was rich with visual cues. The animals were trained to go through one active navigation (A1), one passive navigation (P), and second active navigation (A2) sessions (Fig. 1a). They were trained for $7-10$ days before surgery. A subset $(n=5)$ of animals was trained in an additional passive navigation session (P2). The animals ran 20 laps per each session. The duration of each session was 4-7 min, and there was a $0.5-3-$ min intermission between consecutive sessions. They were placed on a pedestal inside the circular track without disorientation between behavioral sessions.

\section{Unit Recording}

Single units were recorded from the dorsal hippocampus as previously described (Jung et al., 1994). Two tetrodes were implanted (one in each hemisphere) above the hippocampus $(3.8 \mathrm{~mm}$ posterior, $2.0 \mathrm{~mm}$ lateral from bregma, $1.0 \mathrm{~mm}$ ventral from the brain surface) of the well-trained animals under deep sodium pentobarbital anesthesia $(50 \mathrm{mg} / \mathrm{kg})$. After $\geq 1$ week of recovery period from the surgery, tetrodes were lowered into CA1/CA3 cell body layer by manipulating microdrives to obtain unit signals. Unit signals via an FET headstage were amplified 3,000-11,000×, filtered at $0.6-6 \mathrm{kHz}$, digitized at $25 \mathrm{kHz}$, and stored on an IBM-compatible personal computer using custom software. Unit activities were also recorded with the animals placed on the pedestal before and after experimental sessions to examine stability of recorded unit signals. Unstable units were not included in the analyses. EEG was recorded from the opposite hippocampus through one channel of the other tetrode. EEG signals were amplified 300-500×, filtered at $0.1-50 \mathrm{~Hz}$, and digitized at $500 \mathrm{~Hz}$. The animal's location and head direction were monitored by tracking two sets of light-emitting diodes mounted on the headstage at $20 \mathrm{~Hz}$. When recordings were completed, small marker lesions were made and recording locations were verified histologically. The experimental protocol was approved by the Ethics Review Committee for Animal Experimentation of Ajou University School of Medicine.

\section{Analysis}

Single units were isolated by projecting the four channel relative amplitude data two dimensionally, and manually applying boundaries to each subjectively apparent unit cluster using custom software (Xclust, M. Wilson). Spike width was also used as an additional spike waveform characteristic for unit isolation. A spatial firing rate map was constructed for each behavioral session for each unit. The $128 \times 128-\mathrm{cm}$ square containing the circular track was divided into $64 \times 64$ pixels, and the firing rate (total number of spikes divided by total occupancy time) for each pixel was calculated.

"Adaptive binning" was used in this process as described previously (Jung et al., 1994) to optimize the tradeoff between sampling error and resolution. Pixel-by-pixel correlations (r) among firing rates maps were then computed as follows:

$$
r=\frac{\sum\left(f_{i}-\bar{f}\right)\left(g_{i}-\bar{g}\right)}{\sqrt{\sum\left(f_{i}-\bar{f}\right)^{2} \sum\left(g_{i}-\bar{g}\right)^{2}}},
$$

where $f_{i}$ and $g_{i}$ denote firing rates of the $i$-th pixel of two different firing rate maps, and $\overline{\mathrm{f}}$ and $\overline{\mathrm{g}}$ denote firing rates averaged over all pixels. Those pixels that contain 0 occupancy in either firing rate map were excluded from the calculation. The correlation coefficients were transformed to Fisher's z for normalization as the following (Rosner, 1995):

$$
z=0.5[\ln (1+r)-\ln (1-r)] .
$$

Note that Fisher's $\mathrm{z}$ can be $>1$ or $<-1$.

A place field was defined as a group of 10 or more adjoining pixels with the average firing rate of each pixel exceeding three standard deviations above the mean firing rate. Only complexspike cells were included in the analyses concerning place field. Spatial information content per spike was calculated as previously described (Skaggs et al., 1993). The rotation of place-specific firing across different sessions was quantified by measuring a rotation correlation score in a similar manner as in a previous study (Knierim, 2002). Pixel-by-pixel correlation of firing rate maps between two sessions (e.g., A1 vs. P) was calculated while rotating the firing rate map of the second session clockwise by $-177-180^{\circ}$ at $3^{\circ}$ intervals. The rotation angle that produced the highest correlation was taken as the rotation correlation score. Fast-Fourier transforms were performed on EEG data for each behavioral session, and the relative power of $6-9-\mathrm{Hz}$ theta band was calculated. Student's $t$-test and one-way analysis of variance (ANOVA) (repeated measure) were used for statistical comparisons. For rotation correlation scores, Watson-Williams test (circular statistics) was used for statistical comparisons (Batschelet, 1981). A $P$-value of $<0.05$ was used as the criterion for a significant statistical difference. All data are expressed as mean \pm SEM.

\section{RESULTS}

\section{Characteristics of Unit Discharge}

We recorded 60 single units in CA1 and CA3 regions of the dorsal hippocampus in 15 rats during active (A1), passive $(\mathrm{P})$, and second active (A2) navigation sessions (Fig. 1a). Different definitions of "place cells" have been used by different investigators. In the present study, rather than selecting place cells, we included all the neurons that emitted $>20$ spikes in each recording session in the analysis. Thus, after excluding those units $(n=14)$ that emitted $<20$ spikes in any of the three sessions, a total of 46 units (39 complex spike cells and 7 theta cells; Ranck, 1973) were analyzed. Since theta cells also show spatially biased firing (Kubie et al., 1990), both complex spike cells and theta cells were included in the analysis, although same conclusions were obtained when only complex spike cells were analyzed.

The most conspicuous EEG rhythm observed in the hippocampus during an animal movement is $\sim 7 \mathrm{~Hz}$ (theta). In our study, 
a
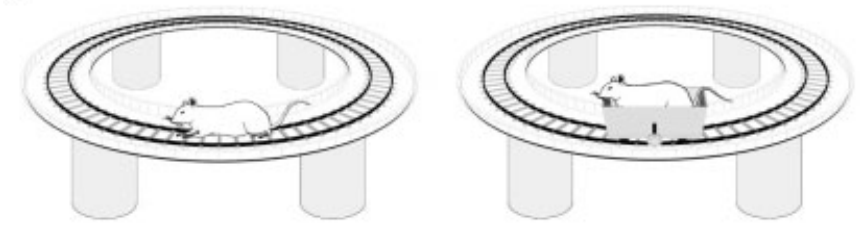

b
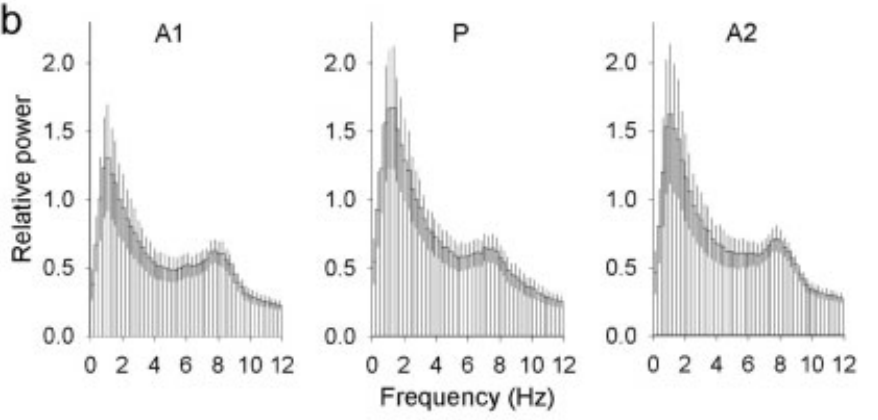

$\mathrm{C}$
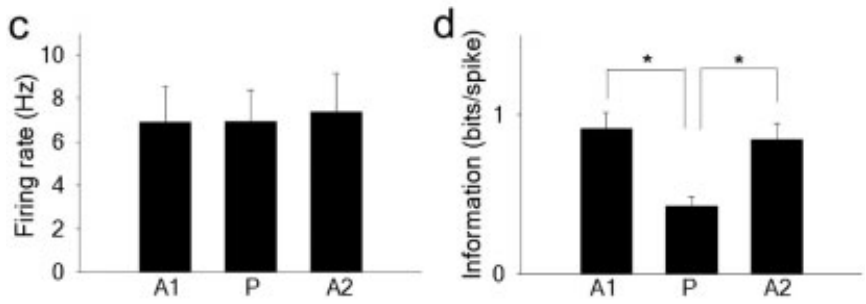

FIGURE 1. Behavioral tasks and unit discharge characteristics. a: Animals alternately went through active and passive navigation sessions on a circular track. Left: active navigation. The animals ran clockwise to obtain water reward at two opposite locations. Right: passive navigation. The animals rode a motorized cart that was controlled by an experimenter. The animals were rewarded at the same locations as in the active navigation condition. b: Averaged EEG power spectrum of the three behavioral sessions $(n=32)$. Bin width is $0.5 \mathrm{~Hz}$. A1 and A2: the first and the second active navigation session. $P$, passive navigation session. c: Mean firing rates of units $(n=$ 46) averaged across the same behavioral sessions. d: Spatial information content $(\mathrm{n}=46) .{ }^{*}$ significant difference $(P<0.001)$.

similar degrees of relative theta EEG power $(6-9 \mathrm{~Hz})$ were observed across A1, $\mathrm{P}$, and A2 sessions (one-way ANOVA, $P>0.05$; Fig. 1b), which is consistent with previous studies that report observation of theta EEG during both active and passive movement of animals (Winson, 1976; Foster et al., 1989; Gavrilov et al., $1995,1996)$. The mean firing rates of the analyzed units $(n=46)$ were $6.9 \pm 1.7,6.9 \pm 1.4$ and $7.4 \pm 1.8 \mathrm{~Hz}$ in $\mathrm{A} 1, \mathrm{P}$, and $\mathrm{A} 2$ sessions, respectively. There was no significant difference in the mean firing rate across the sessions (one-way ANOVA, $P>0.05$; Fig. 1c). However, spatial information content was significantly lower in the P session (one-way ANOVA, $P<0.001$; post-hoc LSD test, $P<0.001$ for both A1-P and P-A2 comparisons), indicating that spatial selectivity of unit discharge is lower under the passive navigation condition (Fig. 1d), which is consistent with the previous report (Gavrilov et al., 1998). The average spatial information contents were $0.91 \pm 0.10,0.43 \pm 0.06$ and $0.84 \pm$ $0.10 \mathrm{bits} /$ spike for $\mathrm{A} 1, \mathrm{P}$, and $\mathrm{A} 2$ sessions, respectively. Because discharges of theta cells are known to depend on rat's motion (Ranck, 1973), the difference in spatial information contents may be attributable to different theta cell activities between active and passive navigation sessions. To rule out this possibility, we excluded theta cells and compared spatial information contents of complex spike cells only $(\mathrm{n}=39)$. They were $1.06 \pm 0.10,0.49 \pm$ 0.06 , and $0.98 \pm 0.11 \mathrm{bits} /$ spike for A1, P, and A2 sessions, respectively. It was significantly lower in the $\mathrm{P}$ session (one-way ANOVA, $P<0.001$; post-hoc LSD test, $P<0.001$ for both A1-P and $\mathrm{P}-\mathrm{A} 2$ comparisons).

\section{Remapping of Place-Specific Firing}

Figure 2a shows representative examples of spatial firing patterns of two complex spike cells (top and middle) and a theta cell (bottom) during A1, P, and A2 sessions. Figure 2b shows pixel-bypixel correlations (transformed to Fisher's $\mathrm{z}$ ) among firing rate maps in A1, P, and A2 sessions of all analyzed units $(\mathrm{n}=46)$. As shown, spatial firing patterns of most units were poorly correlated between $\mathrm{A} 1$ and $\mathrm{P}$ sessions and $\mathrm{P}$ and $\mathrm{A} 2$ sessions, but remained similar between $\mathrm{A} 1$ and $\mathrm{A} 2$ sessions. A one-way ANOVA indicated that a significant difference existed among groups $(P<0.001)$. Post-hoc LSD tests indicated that the correlation between A1 and A2 sessions $(1.15 \pm 0.09)$ was significantly higher than that between both A1-P sessions $(0.23 \pm 0.05, P<0.001)$ and $\mathrm{P}-\mathrm{A} 2$ sessions $(0.18 \pm 0.05, P<0.001)$.

The above result could have arisen due to either remapping or different spatial resolutions of location-specific signal across active and passive navigation conditions. To distinguish between these two possibilities, we compared rotation correlation scores across A1, P, and A2 sessions. As shown in Figure 2d, most data points clustered around 0 for $A 1-A 2$, whereas they were widely dispersed for $\mathrm{A} 1-\mathrm{P}$ and $\mathrm{P}-\mathrm{A} 2$ session pairs. Absolute values of the rotation correlation scores were $65.7 \pm 8.4,17.3 \pm 5.8$ and $65.4 \pm 8.2^{\circ}$ between $\mathrm{A} 1$ and $\mathrm{P}, \mathrm{A} 1$ and $\mathrm{A} 2$, and $\mathrm{P}$ and $\mathrm{A} 2$ sessions, respectively. The rotation correlation score of A1-A2 session pair was significantly different from those of $\mathrm{A} 1-\mathrm{P}$ and $\mathrm{P}-\mathrm{A} 2$ session pairs (Watson-Williams test, $P<0.001$ ), indicating that remapping of spatial firing patterns occurs across active and passive navigation conditions. We also selected only those units that had similar spatial information contents across the three behavioral sessions ( $\mathrm{n}=$ 14 units). Their spatial information contents were $0.17 \pm 0.04$, $0.14 \pm 0.06$ and $0.19 \pm 0.06$ bits $/$ spike for A1, P, and A2 sessions, respectively, which were not significantly different (one-way ANOVA, $P>0.05)$. Their spatial firing rate map correlations (Fig. 2c) were similar to those shown in Figure 2b. The correlation between sessions $\mathrm{A} 1$ and $\mathrm{A} 2(1.16 \pm 0.15)$ was significantly higher than that between $\mathrm{A} 1$ and $\mathrm{P}(0.23 \pm 0.10)$ or $\mathrm{P}$ and $\mathrm{A} 2(0.24 \pm$ 0.14; one-way ANOVA, $P<0.001$; post-hoc LSD tests, $P<$ 0.001 for both comparisons). These results indicate that the low correlation between active and passive navigation sessions is primarily due to remapping of location-specific firing across the two navigation conditions.

If difference in spatial firing across active and passive navigation sessions represents true remapping, one can expect to find those neurons that have place fields in one condition, but not in the other. We tested this possibility by analyzing those complex spike cells that had at least one place field across the three recording 
sessions, regardless of the minimum number of spikes. Of 44 such complex spike cells, $21(47.7 \%)$ had place fields across the three sessions. Fifteen (34.1\%) had place fields in the two active sessions, but not in the passive session. An example of this type of unit is shown in Figure 2e. Two (4.5\%) had place fields in the passive, but not in the active sessions. The rest $(6,13.6 \%)$ showed inconsistent place field activity across the two active sessions. They had place fields in either A1 or A2 session, but not in the other. Thus, 17 of 44 units (38.6\%) had place fields in one navigation condition, but not in the other, further demonstrating remapping of locationspecific firing across the two navigation conditions.

\section{Animal Trajectory}

In the following, we show analysis results that examine whether remapping of location-specific firing across active and passive navigation sessions are attributable to the factors known to influence discharges of hippocampal neurons: differences in movement trajectory, movement speed, head direction, or elevation of the animal (McNaughton et al., 1983; Knierim et al., 2001). For the animal trajectory, we first compared the average lateral head posi-

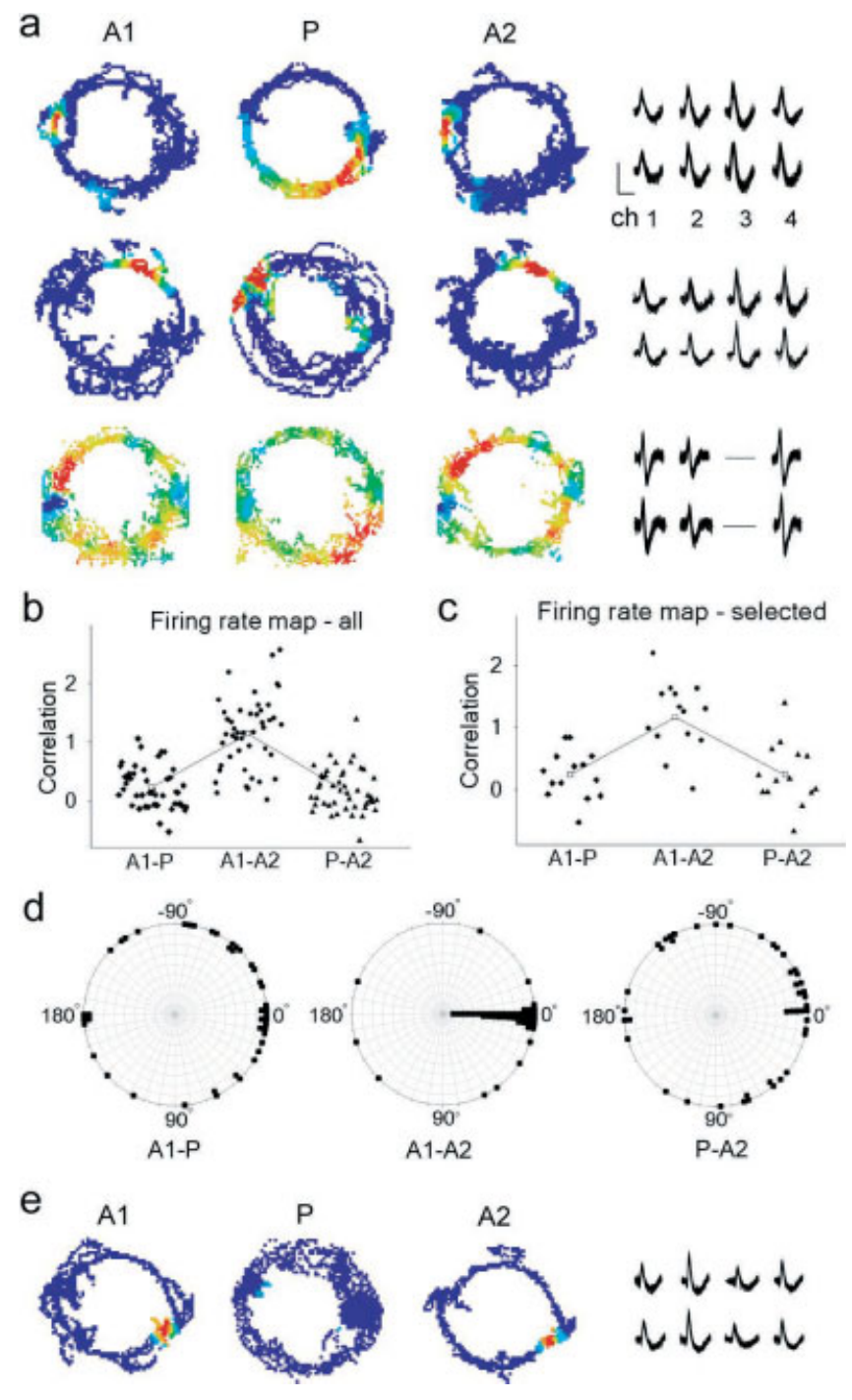

tion along the track across the three recording sessions (Fig. 3a). There were no significant differences among the three sessions ( $\mathrm{n}=32$ sessions, one-way ANOVA, $P>0.05$ ), indicating that lateral deviations of animal head position, on average, are similar across the three sessions. Second, because local variations in the animal trajectory could lead to different spatial firing patterns, we compared spatial distributions of head position (occupancy distributions) on the track across the three sessions. Correlation of occupancy distributions was higher between the two active sessions (z-transformed correlation coefficient: $1.20 \pm 0.06$ ) than between sessions $\mathrm{A} 1$ and $\mathrm{P}(0.34 \pm 0.05)$, or $\mathrm{P}$ and $\mathrm{A} 2(0.38 \pm 0.06$, one-way ANOVA, $P<0.001$, post-hoc LSD tests, $P<0.001$ for both comparisons, Fig. 3b). To test whether the difference in occupancy distribution is responsible for different spatial firing patterns across two navigation conditions, we selected those data that had similar occupancy distributions (i.e., similar trajectories) across the three conditions. As we sequentially removed those data with large differences in occupancy distribution maps, there left a regime of data where occupancy correlations were not significantly different across different behavioral session pairs and $\geq 3$ units were included (3-15 units). Significant remapping of place-specific firing persisted within this regime (one-way ANOVA, $P<$ 0.05 ). In the example shown in Figure $3 c$, only those units with the difference of $<0.3$ in occupancy correlation between any two of the three session pairs were selected. Although occupancy correlations were not significantly different across different behavioral session pairs ( $\mathrm{n}=15$ units, one-way ANOVA, $P>0.05$ ), spatial firing patterns of the selected units were poorly correlated between $\mathrm{A} 1$ and $\mathrm{P}(0.26 \pm 0.12)$ sessions and $\mathrm{P}$ and $\mathrm{A} 2$ sessions $(0.19 \pm$ $0.10)$, but similar between $A 1$ and $A 2$ sessions $(1.34 \pm 0.16)$.

FIGURE 2. Remapping of spatial firing pattern across active and passive navigation conditions. a: Representative examples of complex spike cells (top and middle) and a theta cell (bottom) that remapped their spatial firing patterns across active and passive navigation conditions. Each spatial firing rate map shows the spatial distribution of the firing rate. Red indicates the maximum firing rate that is different for each plot. Top: $26.0,30.9$ and $46.8 \mathrm{~Hz}$ for $\mathrm{A1}, \mathrm{P}$, and $\mathrm{A} 2$ sessions, respectively. Middle: $28.6,1.1$, and $33.8 \mathrm{~Hz}$, respectively. Bottom: $54.2,49.4$, and 51.2, $\mathrm{Hz}$, respectively). Cumulative spike waveforms (30 traces superimposed) recorded through four tetrode channels (ch 1-4) are shown on the right for each cell. Top and bottom rows for each cell correspond to unit signals recorded before and after behavioral sessions, respectively. The theta cell shown was recorded with only three channels leaving out a bad channel (ch 3). Correlations (z-transformed): Top: A1-P: -0.07, A1-A2: 1.48, P-A2: -0.14. Middle: A1-P: -0.08 , A1-A2: 2.19, P-A2: -0.05 . Bottom: A1-P: 0.46, A1-A2: 1.13, P-A2: 0.43. b: Group data. Pixel-by-pixel correlations among firing rate maps were computed and transformed to Fisher's $\mathrm{z}$ for all neurons that emitted $>20$ spikes in each recording session $(n=46)$. Mean correlation coefficients are indicated. c: Pixelby-pixel correlations among firing rate maps of those units that had similar spatial information contents across the three behavioral sessions $(\mathbf{n}=14)$. d: Rotation of place-specific firing. Each dot indicates the amount of rotation of place-specific firing (rotation correlation score) between two sessions. e: An example of complex spike cells that had place fields in only one navigation condition. This unit had a place field in active (A1 and A2), but not in passive (P) navigation session. Maximum firing rates: 10.6, 1.0 and $9.4 \mathrm{~Hz}$ for $\mathrm{A1}, \mathrm{P}$, and $\mathrm{A} 2$ sessions, respectively. Scale bar $=0.5 \mathrm{~ms}$ and $100 \mu \mathrm{V}$. 

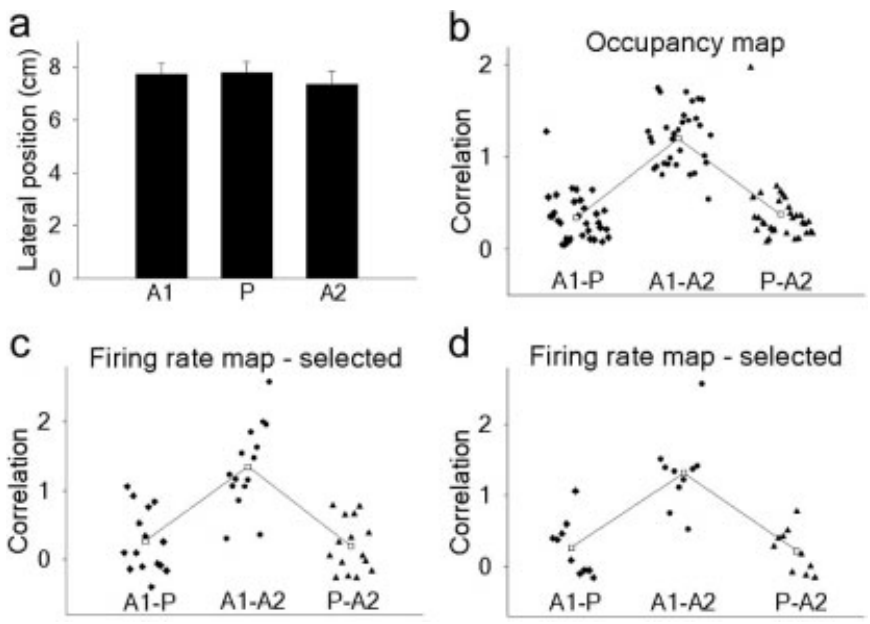

FIGURE 3. Comparison of animal trajectory. a: Average lateral positions of the animals in $A 1, P$, and $A 2$ sessions $(n=32)$. The lateral position was determined by measuring the radial distance of animal position from the inner circle of the track. b: Correlations of spatial distribution of occupancy across the three sessions $(n=32)$. c: Firing rate map correlations of the selected units. Those data that had similar occupancy distributions across A1, P, and A2 sessions were selected $(n=15)$. $d$ : Those units that were recorded in the $A 1$ and $P$ sessions having similar spatial distributions of occupancy near the A1 place field were selected $(n=10)$.

A1-A2 correlation was significantly higher than that of A1-P or P-A2 (one-way ANOVA, $P<0.001$; post-hoc LSD tests, $P<$ 0.001 for both comparisons).

In addition, to test the effect of local variations in the animal trajectory near place fields, we compared animal trajectories near the place field in A1 session. We selected those sections of the track where place fields were located in A1 session, and examined spatial distributions of occupancy during the $P$ session within and outside the A1 place fields. We then selected those sessions in which the animals in the $\mathrm{P}$ session spent $>70 \%$ of the time inside the A1 place field. Spatial firing patterns of the selected units $(n=10)$ were similar only between two active sessions (Fig. 3d). A1-A2 correlation $(1.32 \pm 0.17)$ was significantly higher than that of A1-P $(0.26 \pm 0.12)$ or P-A2 (0.22 \pm 0.1 ; one-way ANOVA, $P<$ 0.001 ; post-hoc LSD tests, $P<0.001$ for both comparisons). Thus, although the animals passively went through the A1 place field $>70 \%$ of the time in the corresponding section of the track, the units did not fire at the same location but mostly elsewhere during the $\mathrm{P}$ session, resulting in a low correlation in the firing rate map between A1 and P sessions. In contrast, units fired at similar locations across $\mathrm{A} 1$ and $\mathrm{A} 2$ sessions.

\section{Movement Speed}

There were no statistical differences in the average movement speed across the three recording sessions ( $n=32$, Fig. 4a), indicating that overall movement speeds were not significantly different. To test local variations in the movement speed, we compared spatial distributions of instantaneous movement speeds on the track across the three sessions. Correlation coefficients of speed distributions (z-transformed) were quite low. They were $-0.004 \pm 0.001,0.000 \pm 0.003$, and $-0.003 \pm 0.001$ for A $1-P$, $\mathrm{A} 1-\mathrm{A} 2$, and $\mathrm{P}-\mathrm{A} 2$ session pairs, respectively (Fig. 4b), which do not vary significantly (one-way ANOVA, $P>0.05$ ). Thus, movement speed distributions were little related across the three sessions.

We also compared variations of the movement speed near the A1 place field. We selected those sessions in which the average movement speeds near A1 place field were similar (differ by $<5$ $\mathrm{cm} / \mathrm{sec}$ ) across A1 and P sessions. The average movement speeds of the selected behavioral sessions near the A1 place field $(\mathrm{n}=17)$ were $12.3 \pm 1.7,12.4 \pm 1.9$ and $14.0 \pm 1.0 \mathrm{~cm} / \mathrm{sec}$ for $\mathrm{A} 1, \mathrm{P}$, and A2 sessions, respectively which do not vary significantly (one-way ANOVA, $P>0.05)$. Spatial firing patterns of the selected units $(\mathrm{n}=17)$ were similar only between two active sessions (Fig. 4c). A1-A2 correlation $(1.26 \pm 0.14)$ was significantly higher than A1-P $(0.15 \pm 0.08)$ or $\mathrm{P}-\mathrm{A} 2$ correlation $(0.08 \pm 0.06$; one-way ANOVA, $P<0.001$; post-hoc LSD tests, $P<0.001$ for both comparisons).

\section{Head Direction}

When we compared spatial distributions of the head direction on the track across the three sessions, there was a higher correlation between the two active sessions than between sessions $\mathrm{A} 1$ and $\mathrm{P}$, or $\mathrm{P}$ and A2 $(\mathrm{n}=32,0.98 \pm 0.07,0.59 \pm 0.04$ and $0.58 \pm 0.03$, respectively; one-way ANOVA, $P<0.001$; post-hoc LSD tests, $P<0.001$ for both comparisons; Fig. 5a). As we removed those data sequentially with large differences in head direction distribution maps, there left a regime of data where head direction correlations were not significantly varied across different behavioral session pairs and $\geq 4$ units were included ( $4-16$ units). Significant remapping of place-specific firing persisted within this regime
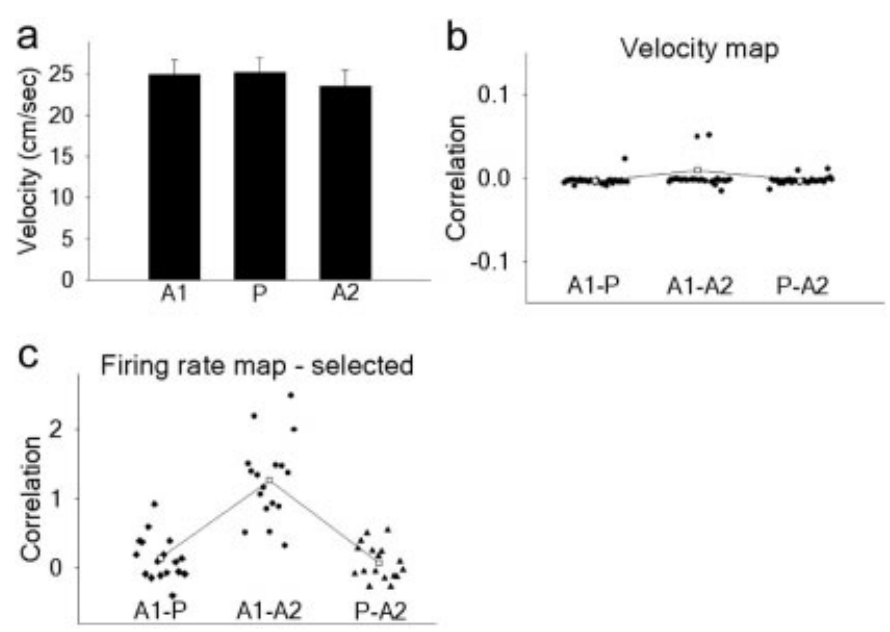

FIGURE 4. Comparison of movement speed. a: Average movement speeds of the animals in A1, P, and A2 sessions $(n=32)$. b: Correlations of spatial distribution of instantaneous movement speeds across the three sessions $(n=32)$. c: Firing rate map correlations of the selected units. Those units that were recorded in the A1 and $P$ sessions having similar movement speeds near the A1 place field were selected $(\mathbf{n}=17)$. 

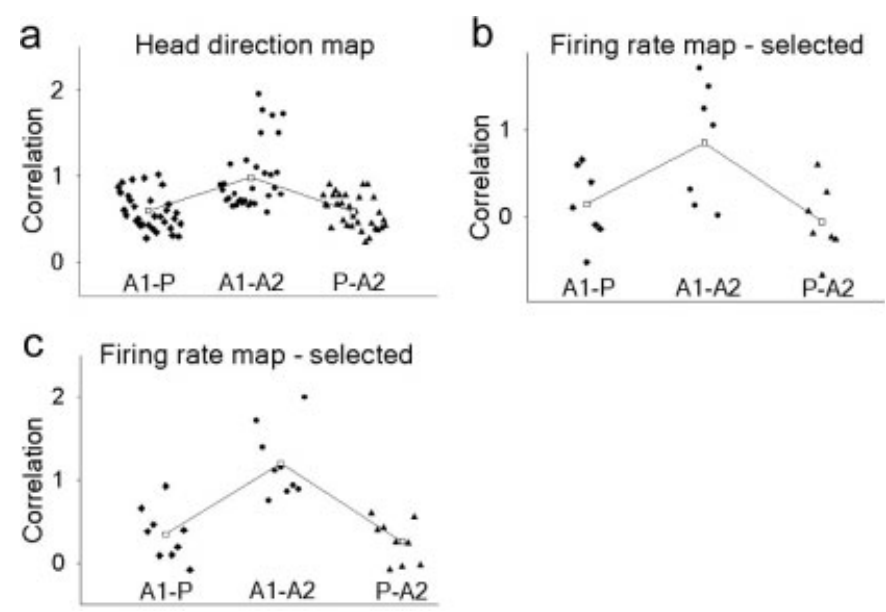

FIGURE 5. Comparison of head direction. a: Correlations of spatial distribution of head direction across $\mathrm{A1}, \mathrm{P}$, and $\mathrm{A} 2$ sessions $(\mathrm{n}=$ 32). b: Firing rate map correlations of the selected units. Those data that had similar head direction distributions across A1, P, and A2 sessions were selected ( $n=7$ units). c: Those units that were recorded in the A1 and P sessions having similar head directions near the A1 place field were selected $(n=9)$.

(one-way ANOVA, $P<0.05$ ). In the example shown in Figure 5b, only those units with the difference of $<0.1$ in head direction correlation between any two of the three session pairs were selected. Although head direction correlations did not vary significantly across different behavioral session pairs ( $\mathrm{n}=7$ units, oneway ANOVA, $P>0.05$ ), spatial firing patterns were more highly correlated between $\mathrm{A} 1$ and $\mathrm{A} 2$ sessions $(0.85 \pm 0.26)$ than between $\mathrm{A} 1$ and $\mathrm{P}(0.14 \pm 0.16)$ or $\mathrm{P}$ and $\mathrm{A} 2$ sessions $(-0.07 \pm 0.16$; one-way ANOVA, $P<0.01$; post-hoc LSD tests, $P<0.01$ for both comparisons).

We also compared variations of head direction near place fields in $\mathrm{A} 1$ session. We selected those sessions in which the average head direction within the selected section of the track were similar (differ by $<10^{\circ}$ ) between $\mathrm{A} 1$ and $\mathrm{P}$ sessions. The difference in average head direction between $\mathrm{A} 1$ and $\mathrm{P}$ sessions near the $\mathrm{A} 1$ place field was $4.6 \pm 2.0^{\circ}$, and that between $\mathrm{A} 1$ and $\mathrm{A} 2$ sessions was $-3.7 \pm$ $4.5^{\circ}$ for the selected sessions $(n=9)$. These relative head directions were not significantly different from each other $(t$-test, $P>0.05)$. Again, spatial firing patterns of the selected units $(n=9)$ were similar only between two active sessions (Fig. 5c). A1-A2 correlation $(1.20 \pm 0.14)$ was significantly higher than A1-P $(0.35 \pm$ $0.10)$ or P-A2 correlation ( $0.26 \pm 0.09$; one-way ANOVA, $P<$ 0.001; post-hoc LSD tests, $P<0.001$ for both comparisons).

To test the combined effect of variations in animal behavior, we quantified how much variance in firing rate map correlations could be accounted for by variations in animal trajectory, movement speed, and head direction. Firing rate map correlations of all analyzed units across all session pairs (Fig. 2b) were subject to a multiple regression analysis for this purpose. The coefficients of occupancy, speed, and head direction map correlation served as independent variables. The adjusted $R^{2}$ of the multiple regression was 0.27 , indicating that $27 \%$ of variance can be predicted from the values of the three independent variables. When the navigation condition (i.e., A1-P, A1-A2, and P-A2) was added as an additional independent variable to the regression, they accounted for $51 \%$ of the total variance of firing rate map correlations altogether (i.e., adjusted $R^{2}=0.51$ ). These results indicate that variations in animal behavior, even when considered together, cannot fully account for the observed remapping of place-specific firing.

\section{Unit Firing at Different Elevation of the Rat}

The head of the animal was elevated $\sim 4.5 \mathrm{~cm}$ higher in the $\mathrm{P}$ session than other sessions because of the height of the motorized cart. To examine whether the observed remapping could be due to different heights (Knierim et al., 2001), we recorded 11 cells from four rats in two active navigation sessions with two different heights. The circular track was elevated by $4.5 \mathrm{~cm}$ (equivalent to passive navigation condition) in the second session. As shown by an example in Figure 6, similar firing patterns were observed across the two sessions. The average correlation was $1.03 \pm 0.21(\mathrm{n}=11$ units), which was significantly greater than the correlation between $\mathrm{A} 1$ and $\mathrm{P}$ sessions $(0.23 \pm 0.05, t$-test, $P<0.01)$. These results indicate that the observed remapping across active and passive navigation sessions is not attributable to the difference in the elevation of the animals.

\section{Stable Representation of Space Across Repeated Passive Navigation Sessions}

The remapping observed in the P session might have been a random process that resulted from animals paying little attention to the external environment during the P session. Different levels of attention could lead to different levels of modulatory signals to the hippocampus, which may in turn induce changes in hippocampal unit firing. Alternatively, spatial information is represented stably but differently in the hippocampus during the P session. These alternative possibilities were examined by recording cells in repeated passive navigation sessions. A subset of hippocampal neurons $(n=9$ out of 46$)$ was recorded throughout the first active (A1), first passive (P1), second active (A2), and second passive (P2) navigation sessions from four rats. As shown in Figure 7, spatial firing patterns between the two active ( $\mathrm{A} 1$ and $\mathrm{A} 2$ ) as well as the two passive sessions (P1 and P2) were maintained stably. Both A1-A2 $(1.58 \pm 0.25)$ and P1-P2 correlations $(0.49 \pm 0.08)$ were significantly greater than zero ( $t$-test, $P<0.001$ and $P<0.01$, respectively), and they were significantly higher than other correlations (one-way ANOVA, $P<0.001$; post-hoc LSD tests, A1-A2 vs. other active-passive correlations: $P<0.001, \mathrm{P} 1-\mathrm{P} 2$ vs. other active-passive correlations: $P<0.05$ ). These results demonstrate that spatial information under passive navigation condition is stably represented in the hippocampus.

\section{Partial Remapping}

Firing rate map correlations between $\mathrm{A} 1$ and $\mathrm{P}$ sessions and $\mathrm{P}$ and A2 sessions were significantly larger than 0 ( $t$-test, $P<0.001$ and $P<0.01$, respectively), suggesting that the observed remapping across the active and passive sessions was not a random process. To test this possibility further, we randomly rotated firing rate 


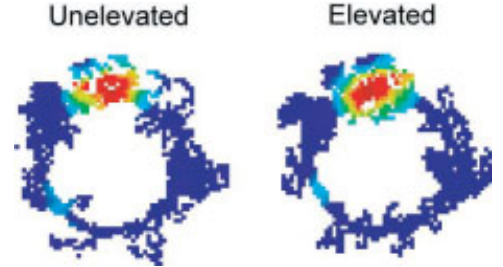

FIGURE 6. Comparison of unit firing at different elevation of the rat in the active condition. This example shows unit firing at two different heights of the track. The correlation (z-transformed) between the two firing rate maps is 1.90. Maximum firing rates: 31.1 and $29.1 \mathrm{~Hz}$, respectively.

maps of $\mathrm{A} 1$ session $\left(\mathrm{A} 1^{\prime}\right)$ and computed their correlations with the original A1 firing rate maps. The correlation (z-transformed) between the two conditions was $0.01 \pm 0.05$, which was not significantly different from $0(t$-test, $P>0.05)$ and significantly lower than the A1-P correlation ( $t$-test, $P<0.01$; Fig. 8b). Some neurons maintained their spatial firing patterns across active and passive navigation conditions (Fig. 8a), suggesting that the above results may be due to mixed responses (some units remap, whereas others maintain their spatial firing patterns). To test this possibility, we calculated rotation correlation scores across A1 and P sessions (Fig. 2d), and then divided the units into "maintained" and "remapped" categories. If rotation correlation score was within \pm

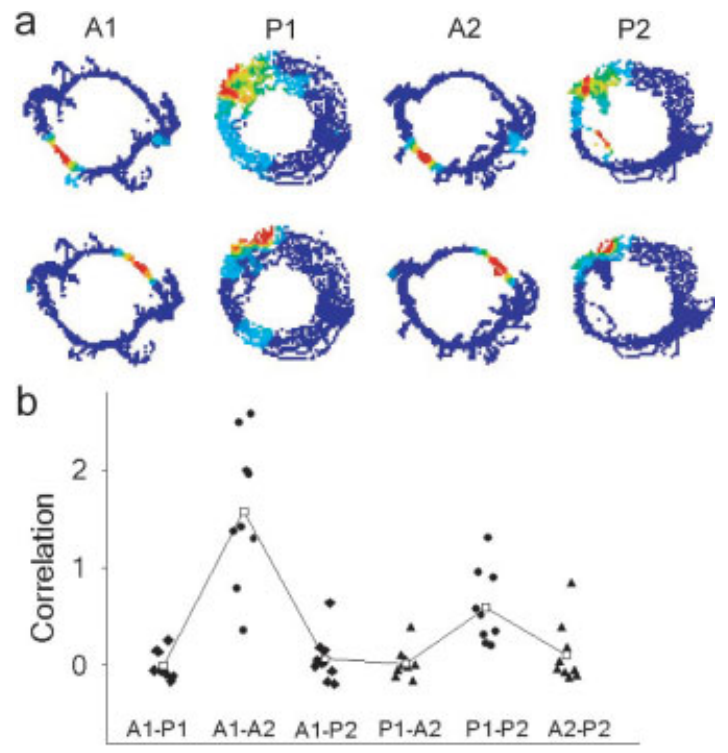

FIGURE 7. Stable representation of spatial information across repeated passive navigation sessions. a: Two examples of unit firing across two active and passive navigation sessions. Maximum firing rates: top: $26.3,9.1,33.3$ and $8.5 \mathrm{~Hz}$; bottom: $11.4,4.3,16.9$, and 2.9 $\mathrm{Hz}$ (left to right). Correlations (z-transformed): Top: A1-A2: 1.99, P1-P2: 1.31, A1-P1: -0.08, A1-P2: -0.17, P1-A2: -0.03, A2-P2: -0.14. Bottom: A1-A2: 2.57, P1-P2: 0.90, A1-P1: -0.16, A1-P2: -0.06, P1-A2: -0.16, A2-P2: -0.05. b: Group data. Pixel-by-pixel correlations among firing rate maps are shown for nine units. Mean correlation coefficients are indicated.

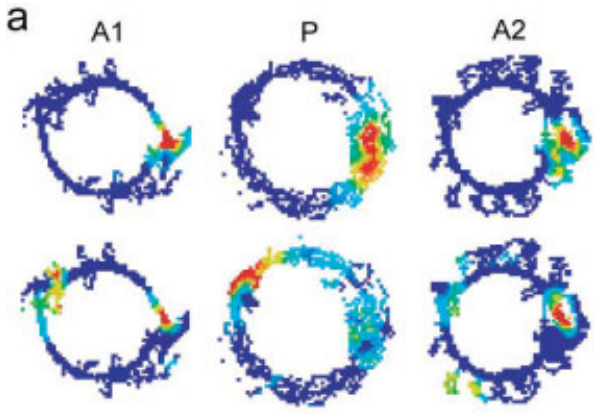

b

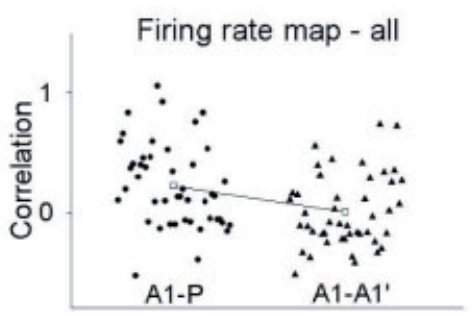

FIGURE 8. Partial remapping. a: Simultaneously recorded units with mixed responses. One unit maintained, and the other remapped their spatial firing patterns across active and passive navigation conditions. Maximum firing rates: Top: $29.4,22.0$, and $36.7 \mathrm{~Hz}$. Bottom: 3.4, 12.0 and $3.7 \mathrm{~Hz}$ (left to right). Correlations (z-transformed): Top: A1-P: 0.76, A1-A2: 1.85, P-A2: 0.66. Bottom: A1-P: -0.39, A1-A2: 1.47, P-A2: - 0.26. b: Correlations between A1 and P firing rate maps, and between $A 1$ and randomly rotated $A 1$ (A1') firing rate maps.

$9^{\circ}$, the unit was defined to have "maintained" place-specific firing across the two sessions. Otherwise, units were defined to have "remapped" place-specific firing. There were nine "maintained" (19.6\%) and 37 "remapped" (80.4\%) units. The chance for a unit to have maintained place-specific firing after random rotation is only $5 \%$, and the probability for 46 units to have 9 or more maintained units after random rotation is $<0.001$, indicating that a significant number of units maintained place-specific firing across $\mathrm{A} 1$ and $\mathrm{P}$ sessions. Similar results were obtained with the analyses concerning P and A2 sessions (data not shown).

We then examined whether simultaneously recorded units behaved in the same way (maintenance vs. remapping of spatial firing patterns) across active and passive navigation conditions. Of the nine units that maintained their firing patterns across $\mathrm{A} 1$ and P sessions (i.e., rotation correlation score was within $\pm 9^{\circ}$, Fig. 2d), five were simultaneously recorded with one or more units. These five units were recorded in five separate recording sessions; in other words, each unit was recorded with one or more units that showed remapping of place-specific firing across $\mathrm{A} 1$ and $\mathrm{P}$ sessions. Figure $8 \mathrm{a}$ shows such an example. As shown, one neuron remapped, but the other neuron maintained placespecific firing across $\mathrm{A} 1$ and $\mathrm{P}$ sessions. These results show that some neurons maintained, but at the same time, others remapped their spatial firing patterns across active and passive navigation conditions (i.e., partial remapping). 


\section{DISCUSSION}

\section{Role of Active Movement in Place-Specific Firing of Hippocampal Neurons}

The results of this study show that spatial information is represented differently across active and passive navigation conditions in an identical environment. The distal visual cues were identical under the two navigation conditions and the animals were not disoriented nor removed from the recording room. The observed remapping cannot be explained by the difference in movement trajectory, velocity, head direction, or height of the animal. The remapping did not occur due to an unknown time-dependent variable either, because spatial firing was stably maintained across repeated active and passive navigation sessions. Care was taken to maintain the variation in local cues (tactile and olfactory) in a minimal level. The texture or odor of the moving cart was not likely a factor in location-specific firing on the track, because it was not stationary but moved along the track. We cannot completely rule out the possibility that potential variations in the texture or odor on the track contributed to the observed remapping of placespecific firing. However, results from previous studies suggest that this is not a likely possibility. In a number of studies using a cylinder or a square box, the floor of the arena was covered with a new sheet of paper before each recording session; i.e., potential local cues on the floor were different across different recording sessions. In such cases, as long as distal cues remained the same, place specific firing was maintained relative to the distal cues (e.g., Muller and Kubie, 1987). Perhaps local cues, if they exist, and distal cues are associated so that either one can drive place-specific firing at the same location. The absence of local cues (such as in the $P$ condition) would not alter spatial firing of hippocampal neurons as long as distal cues are intact. Regarding potential variations in odor cues on the track in particular, they must have been strong and consistent if they have influenced unit firing during active sessions, because similar place-specific firing was observed across repeated active sessions. If this is the case, it is likely that the odor cues were available to the animal during the passive navigation condition, because the animal was mostly sticking its head out of the moving cart. Finally, the relative power of conspicuous theta EEG and average firing rate were similar between the two conditions, suggesting that information was similarly processed under these conditions. Combined, external sensory information and internal path integration signals would lead to the same spatial computation under the two navigation conditions. In this circumstance, if the hippocampus concerns only the spatial computation regardless of the mode of navigation, then similar spatial firing patterns should be observed across the two navigation conditions. Even when considering different patterns of sensory input to the hippocampus across the two navigation modes, place fields should be found at similar locations with somewhat different spatial resolutions or some drift. However, instead, place-specific firing remapped across the two navigation modes in our study. These results strongly suggest that the difference in the liberty of move- ment between the two navigation conditions is responsible for the observed remapping.

\section{Neural Mechanisms Underlying Remapping}

What is the neural mechanism that leads to different representations of space across active and passive navigation conditions? Neural signals related to voluntary movement, such as motor command signals, efference copy or proprioceptive feedback signals may play important roles in determining hippocampal neuronal activity. Alternatively, active and passive navigations may be represented as separate behavioral tasks. Previous studies have shown that spatial firing patterns of hippocampal neurons in the same environment may be altered depending on behavioral tasks (Markus et al., 1995; Kobayashi et al., 1997). It is possible that brain structures such as the prefrontal cortex play important roles in this process. Prefrontal cortex is known to encode "rules" of behavioral tasks (Winocur and Moscovitch, 1990; Jung et al., 1998; Wallis et al., 2001) and it is heavily interconnected with the hippocampal formation (Witter, 2003). Additional work is needed to identify neural mechanisms underlying different representations of space across active and passive navigation conditions.

\section{Performance Under Different Navigation Conditions}

In our daily experience with automobiles, a passive navigator (passenger) has much more trouble than an active navigator (the driver) in subsequent spatial navigation in an unfamiliar region. Following passive navigation in a simulated three-dimensional environment, the subjects' performance during the subsequent active navigation (test trials) was significantly lower compared to those who underwent prior active navigation (Peruch et al., 1995). Our results suggest that the poor performance in test trials following passive navigation may be due to different representations of space between the two navigation conditions. Assuming that spatial information is separately represented across active and passive navigation conditions in the human hippocampus as in the rat, it would be more difficult to use spatial information acquired in one condition (e.g., passive navigation) on the other navigation condition (e.g., active navigation). Furthermore, the spatial information content of unit activity was lower under the passive navigation condition, suggesting that external space is represented with lower resolution during passive navigation. This would lead to inferior spatial learning during passive navigation compared to active navigation. It should be noted, however, that the experimental settings in our study are quite different from those in the human navigation study (Peruch et al., 1995). It is also unclear whether or not humans and rats share common spatial navigation strategies or common neural mechanisms for representation of spatial information. Additional work is needed to resolve these matters.

\section{Interplay Between Spatial and Nonspatial Factors}

The scope of hippocampal memory functions has long been debated. Since the proposal by O'Keefe and Nadel (1978), the 
cognitive map theory has maintained that the hippocampus primarily concerns spatial memory. Others argue that the hippocampus subserves broader memory functions (declarative, relational or episodic memory) and that spatial memory is just one aspect of hippocampal memory functions (e.g., Squire, 1987; Eichenbaum et al., 1999). According to the latter argument, the results of our study can be interpreted as reflecting qualitatively different episodes that occur in the same place while running or riding. On the other hand, although behavioral structures were rather homogenous on the track except at the two reward locations, strong location-specific firing was observed under both navigation conditions. This observation is consistent with the possibility that spatial information processing is an inherent function of the hippocampus. Whereas a number of studies have shown nonspatial correlates (for review, see Eichenbaum et al., 1999; Redish 1999, 2001), placespecific firing is perhaps the most conspicuous and consistent feature of hippocampal neuronal activity, at least in the rat. These observations suggest that hippocampal unit activity is an outcome of interactions between spatial and nonspatial factors. Then how do they interact? Two different views have been proposed regarding this matter. O'Keefe and Nadel (1978) have proposed that the hippocampus contains a spatial map in which representations of objects experienced in the environment are ordered within a framework generating a unitary space. On the other hand, several investigators (McNaughton et al., 1996; Redish, 1999; Sharp, 1999; Jeffery and Anderson, 2003) have proposed that the hippocampus stores multiple spatial maps of the same environment depending on behavioral contexts. How can we reconcile these two different views? First, they are not mutually exclusive; the hippocampus may store multiple spatial maps depending on behavioral contexts, with each map providing a unitary spatial framework where memories about various events in the corresponding behavioral context are to be stored. Second, although these views are different at the conceptual level, they do not necessarily require separate neural mechanisms. Partial remapping (e.g., Knierim, 2002; Anderson and Jeffery, 2003; present study) suggests that hippocampal place-specific firing is not an outcome of strong attractor dynamics (weak external input connections and strong internal connections), but rather, it is under relatively strong modulation by incoming sensory information. Assuming that hippocampal unit firing is largely determined by relative weights of different sources of information impinging on a given neuron, most hippocampal units would remap their firing patterns under two largely different conditions, whether the difference is two different behavioral contexts in the same environment or an occurrence of a certain event at a certain location under only one condition. As such, the two conceptual arguments may not be different in terms of neural network dynamics. Perhaps one role of the hippocampus is to associate spatial information with nonspatial information that may be separately provided by the medial and lateral entorhinal cortex, respectively (Quirk et al., 1992; Otto et al., 1996; Hargreaves et al., 2002; Knierim, 2003). This would, depending on the experimental design, appear as storing multiple maps for different behavioral contexts or providing a single spatial framework within which objects and events are to be stored.

\section{Acknowledgments}

The authors thank S.I. Wiener for helpful comments on the manuscript.

\section{REFERENCES}

Anderson MI, Jeffery KJ. 2003. Heterogeneous modulation of place cell firing by changes in context. J Neurosci 23:8827-8835.

Batschelet E. 1981. Circular statistics in biology. London: Academic Press.

Eichenbaum H, Dudchenko P, Wood E, Shapiro M, Tanila H. 1999. The hippocampus, memory, and place cells: is it spatial memory or a memory space? Neuron 23:209-226.

Foster TC, Castro CA, McNaughton BL. 1989. Spatial selectivity of rat hippocampal neurons: dependence on preparedness for movement. Science 244:1580-1582.

Gavrilov VV, Wiener SI, Berthoz A. 1995. Enhanced hippocampal theta EEG during whole body rotations in awake restrained rats. Neurosci Lett 197:239-241.

Gavrilov VV, Wiener SI, Berthoz A. 1996. Whole-body rotations enhance hippocampal theta rhythmic slow activity in awake rats passively transported on a mobile robot. Ann NY Acad Sci 781:385-398.

Gavrilov VV, Wiener SI, Berthoz A. 1998. Discharge correlates of hippocampal complex spike neurons in behaving rats passively displaced on a mobile robot. Hippocampus 8:475-490.

Hargreaves EL, Fu L, Knierim JJ. 2002. Spatial tuning of cortical inputs to the hippocampus: medial vs. lateral. Soc Neurosci Abs 28:477.12.

Jeffery KJ, Anderson MI. 2003. Dissociation of the geometric and contextual influences on place cells. Hippocampus 13:868-872.

Jung MW, McNaughton BL. 1993. Spatial selectivity of unit activity in the hippocampal granular layer. Hippocampus 3:165-182.

Jung MW, Wiener SI, McNaughton BL. 1994. Comparison of spatial firing characteristics of units in dorsal and ventral hippocampus of the rat. J Neurosci 14:7347-7356.

Jung MW, Qin Y, McNaughton BL, Barnes CA. 1998. Firing characteristics of deep layer neurons in prefrontal cortex in rats performing spatial working memory tasks. Cereb Cortex 8:437-450.

Knierim JJ. 2002. Dynamic interactions between local surface cues, distal landmarks, and intrinsic circuitry in hippocampal place cells. J Neurosci 22:6254-6264.

Knierim JJ. 2003. Hippocampus and memory: can we have our place and fear it too? Neuron 37:372-374.

Knierim JJ, McNaughton BL. 2001. Hippocampal place-cell firing during movement in three-dimensional space. J Neurophysiol 85:105-116.

Kobayashi T, Nishijo H, Fukuda M, Bures J, Ono T. 1997. Task-dependent representations in rat hippocampal place neurons. J Neurophysiol 78:597-613.

Kubie JL, Muller RU, Bostock E. 1990. Spatial firing properties of hippocampal theta cells. J Neurosci 10:1110-1123.

Markus EJ, Barnes CA, McNaughton BL, Gladden VL, Skaggs WE. 1994. Spatial information content and reliability of hippocampal CA1 neurons: effects of visual input. Hippocampus 4:410-421.

Markus EJ, Qin YL, Leonard B, Skaggs WE, McNaughton BL, Barnes CA. 1995. Interactions between location and task affect the spatial and directional firing of hippocampal neurons. J Neurosci 15:7079-7094.

McNaughton BL, Barnes CA, O'Keefe J. 1983. The contributions of position, direction and velocity to single unit activity in the hippocampus of freely-moving rats. Exp Brain Res 52:41-49.

McNaughton BL, Barnes CA, Gerrard JL, Gothard K, Jung MW, Knierim JJ, Kudrimoti H, Qin Y, Skaggs WE, Suster M, Weaver KL. 1996. Deciphering the hippocampal polyglot: the hippocampus as a path integration system. J Exp Biol 199:173-185. 
Muller RU, Kubie JL. 1987. The effects of changes in the environment on the spatial firing of hippocampal complex-spike cells. J Neurosci 7:1951-1968.

O'Keefe J, Dostrovsky J. 1971. The hippocampus as a spatial map. Preliminary evidence from unit activity in the freely-moving rat. Brain Res 34:171-175.

O'Keefe J, Conway DH. 1978. Hippocampal place units in the freely moving rat: why they fire where they fire. Exp Brain Res 31:573-590.

O'Keefe J, Nadel L. 1978. The hippocampus as a cognitive map. Oxford: Clarendon Press.

O’Keefe J, Speakman A. 1987. Single unit activity in the rat hippocampus during a spatial memory task. Exp Brain Res 68:1-27.

Otto T, Ding C, Cousens G, Schiller K. 1996. Effects of lateral vs. medial entorhinal cortex aspiration on the acquisition of odor-place associations. Soc Neurosci Abs 22:443.12.

Peruch P, Vercher JL, Gauthier GM. 1995. Acquisition of spatial knowledge through visual exploration of simulated environments. Ecol Psychol 7:1-20.

Quirk GJ, Muller RU, Kubie JL. 1990. The firing of hippocampal place cells in the dark depends on the rat's recent experience. J Neurosci 10:2008-2017.

Quirk GJ, Muller RU, Kubie JL, Ranck JB Jr. 1992. The positional firing properties of medial entorhinal neurons: description and comparison with hippocampal place cells. J Neurosci 12:1945-1963.

Ranck JB Jr. 1973. Studies on single neurons in dorsal hippocampal formation and septum in unrestrained rats. I. Behavioral correlates and firing repertoires. Exp Neurol 41:461-531.

Redish AD. 1999. Beyond the cognitive map: from place cells to episodic memory. Cambridge, MA: MIT Press.
Redish AD. 2001. The hippocampal debate: are we asking the right questions? Behav Brain Res 127:81-98.

Rosner B. 1995. Fundamentals of biostatistics. Belmont, CA: Duxbury Press.

Save E, Nerad L, Poucet B. 2000. Contribution of multiple sensory information to place field stability in hippocampal place cells. Hippocampus 10:64-76

Sharp PE. 1999. Complimentary roles for hippocampal versus subicular/ entorhinal place cells in coding place, context, and events. Hippocampus 9:432-443.

Skaggs WE, McNaughton BL, Gothard KM, Markus EJ. 1993. An information-theoretic approach to deciphering the hippocampal code. In: Hanson SJ, Cowan JD, Giles CL, editors. Advances in neural information processing systems. San Mateo, CA: Morgan Kaufmann. p $1030-1037$.

Song EY, Jung MW. 2002. Remapping of place fields across active and passive navigation conditions. Soc Neurosci Abs 27:677.18.

Squire LR. 1987. Memory and brain. Oxford: Oxford University Press.

Wallis JD, Anderson KC, Miller EK. 2001. Single neurons in prefrontal cortex encode abstract rules. Nature 411:953-956.

Winocur G, Moscovitch M. 1990. Hippocampal and prefrontal cortex contributions to learning and memory: analysis of lesion and aging effects on maze learning in rats. Behav Neurosci 104:544-551.

Winson J. 1976. Hippocampal theta rhythm. I. Depth profiles in the curarized rat. Brain Res 103:57-70.

Witter MP. 2003. Organization of cortico-hippocampal networks in rats related to learning and memory. In: Ono T, Matsumoto G, Llinas RR, Berthoz A, Norgen R, Nishijo H, Tamura R, editors. International Congress Series 1250. Cognition and emotion in the brain. Amsterdam: Elsevier. p 131-145. 